\title{
Development of research methods for mulberry silkworm disease- nuclear polyhedrose
}

\begin{abstract}
Nuclear polyhedrosis, that is jaundice, is distinguished among other mulberry silkworm diseases by its pathogenicity. It is a powerful viral, hereditary disease of mulberry silkworm, that results in mass destruction of mulberry silkworm. Hence, early diagnostics of resistance of silkworm breeds and hybrids to the diseasepolyhedrose is possible by the application of modern achievements of biochemistry and molecular genetics. More efficient sampling of worm individuals by reliable and express testing is very urgent for further selection activity.

The goal of our research is control of productivity, in particular preparation of high quality and healthy grain, and thus prevention of decrease of cocoon productivity by diseases. Silkworm feeding was performed by strict observance of the fixed agro rules. Infected worms were obtained by the method of induction, hemolymph was isolated from diseased worms and was collected in Eppendorf tube, was washed and pure polyhedrons were obtained. Various titers of polyhedron suspensions were prepared from hemolymph which was sprayed on the leaf which was given to the silkworms.

As a result of experiments the concentration of polyhedron suspension was determined at which silkworms were infected slightly but it managed to make cocoon, to pass the process of metamorphosis and lay grain. Worms which escaped death made cocoon and the grain was prepared from those worms. This grain passed through testing for a disease and was used in further feeding. Similarly, polyhedrose virus was obtained from the grain infected by polyhedrose virus. The method developed by us enables us to receive polyhedrons of high purity. Weakly diseased grain was used for feeding silkworm, which at further feeding and testing will give breeds resistant to the disease nuclear polyhedrose.
\end{abstract}

Keywords: silkworm, hemolymph, disease, polyhedrose
Volume 4 Issue 3 - 2017

\author{
Nargiz Baramidze,' Giorgi Gigolashvili,, Maia \\ Khutsishvili, ${ }^{2}$ Zoia Tskaruashvili, ${ }^{2}$ Shorena \\ Kharatishvili' \\ 'Research Center of the Ministry of Agriculture, Georgia \\ ${ }^{2}$ Agrarian university, Georgia
}

Correspondence: Nargiz Baramidze, Senior Specialist at the Research and Research Center of the Ministry of Agriculture, Georgia, Email n.baramidze@agruni.edu.ge

Received: October 28, 2017 | Published: December 20, 2017

\section{Introduction}

Nuclear polyhedrosis, that is jaundice, is distinguished among other mulberry silkworm diseases by its pathogenicity. It is a powerful viral, hereditary disease of mulberry silkworm that results in mass destruction of mulberry silkworm. Polyhedrose is spread in all silk manufacturer countries and incurs the losses to sericulture more than any infectious diseases of mulberry silkworm. Viruses which insite this disease are characterized by hexagonal form crystalline formations, that is polyhedrons, detected in the hemolymph (blood) of diseased warms by microscope, but in the grain phase polyhedrons can't be fixed even by a microscope. The period from the moment of infection till its exposure (6-8 days) is a hidden, latent period; length of this period depends on worm premise temperature, humidity and feed quality. ${ }^{1}$

The significant task of sericulture is heightening of resistance of mulberry silkworm breeds and hybrids to unfavorable environment conditions and diseases. Mulberry silkworm metabolism is its poikilothermal property, because of which it depends greatly on environment conditions, especially on temperature, which determines the progress of biochemical processes and it can result in changes of biotechnological indices, especially decrease of worm viability.

In distinct from other insects, mulberry silkworm depends more on the impact of environment factors, rearing and various approaches in artificial selection carried out by researchers. Metabolic processes of mulberry silkworm are significantly changed at the alteration of temperature, which in its turn conditions changes in growthdevelopment, voltinity and other vital processes. Irrespective of the fact that domestication and rearing of mulberry silkworm has a rather long history, it is still the subject of interest of study and investigation of researchers. At the same time, the silkworm, by its character is the most significant object for development of theoretical and practical problems of biology, genetics and selection. Study of historic development, hybridization, rearing and selection principles of silkworm is very urgent to enable us to control its growthdevelopment and productivity. ${ }^{2}$

One of the major tasks of contemporary genetics and selection in sericulture is creation of highly productive breeds and hybrids. Traditional methods of assessment of efficiency of various breeds and hybrid combinations require lengthy and hard, labor-consuming experimental feedings. Hence, early diagnostics of resistance of silkworm breeds and hybrids to the disease-polyhedrose is possible by the application of modern achievements of biochemistry and molecular genetics. More efficient sampling of worm individuals by reliable and express testing is very urgent for further selection activity. ${ }^{3}$

\section{Methodology}

The goal of our research is control of productivity, in particular preparation of high quality and healthy grain, and thus prevention 
of decrease of cocoon productivity by diseases. Silkworm feeding was performed by strict observance of the fixed agro rules. Infected worms were obtained by the method of induction, hemolymph was isolated from diseased worms and was collected in Eppendorf tube, was washed and pure polyhedrons were obtained. Various titers of polyhedron suspensions were prepared from hemolymph which was sprayed on the leaf which was given to the silkworms.

Hemolymph was centrifuged $10000 \mathrm{~g}$, for 2 minutes. Residue that contained worm hemolymph cells (formed elements) and polyhedrons were washed 3-times in distilled water. To receive pure virus polyhedrons the obtained residue was dispersed in distilled water and was spread on glycerin concentrate gradient $(25,50$ and $80 \%$ glycerin layers in a centrifuge tube). Tubes were centrifuged at $3000 \mathrm{rev} / \mathrm{min}(1200 \mathrm{~g})$ for $20 \mathrm{~min} .2$ fractions (Figure1) were obtained, which were called, correspondingly, the light (at the border of 25 and 50\%- glycerin layers) and heavy (at the border of 50 and $80 \%$-glycerin layers) fractions. Fractions were collected and washed to remove glycerin. ${ }^{4}$

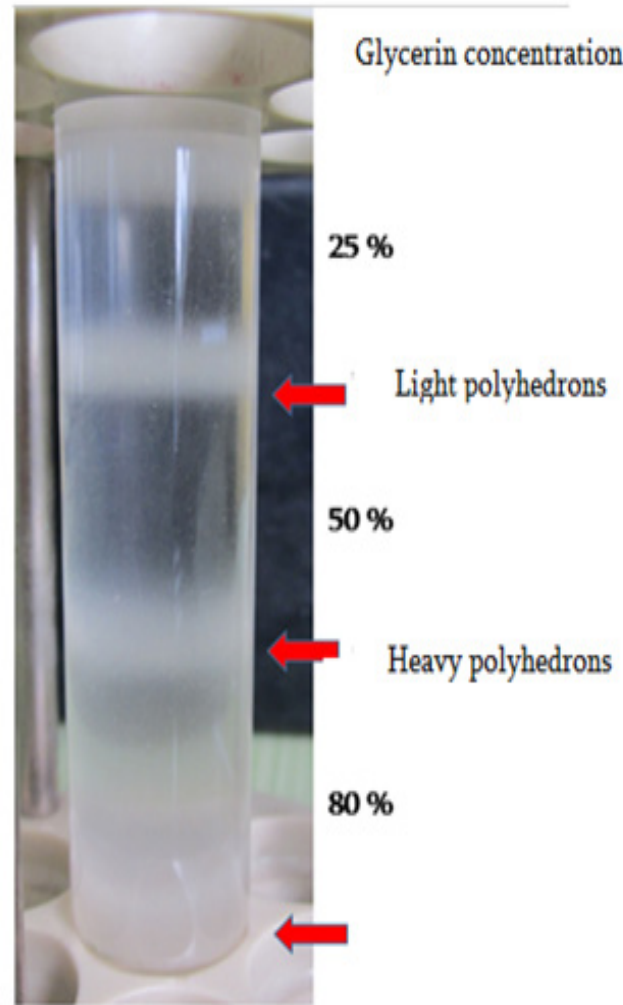

Figure I Polyhedron fractions after centrifugation in glycerin concentration step-wise gradient.

\section{Results}

As a result of experiments the concentration of polyhedron suspension was selected at the application of which the worms are diseased to a level, when they are able to make cocoon and lay grain. With this in view the polyhedron suspension of the above referred concentration was sprayed on the leaf and given to the worm in the morning on an empty stomach. In some versions disease declared itself in 2-3 days, while the silkworm infected by nuclear polyhedrose virus was brought to the stage of a moth and after papillonage the grain infected by nuclear polyhedrose virus was obtained.
As a result of experiments the concentration of polyhedron suspension was determined at which silkworms were infected slightly but it managed to make cocoon, to pass the process of metamorphosis and lay grain. Worms which escaped death made cocoon and the grain was prepared from those worms. This grain passed through testing for a disease and was used in further feeding. Similarly, polyhedrose virus was obtained from the grain infected by polyhedrose virus. Electronic microscope proved that the light fraction, there are also cell fractions and other mechanical admixes (Figure1) together with the small size polyhedrons. Polyhedrons of the heavy fraction were purified from admixes at maximum (Figure2)..$^{5-7}$

Figure 2 shows well the polyhedrons (marked by arrows) and other coarse admixes. Figure 3 shows small particles around polyhedron, probably dissociated viruses. Figure 4 Fixed polyhedron virus particles in the form of bacillus.

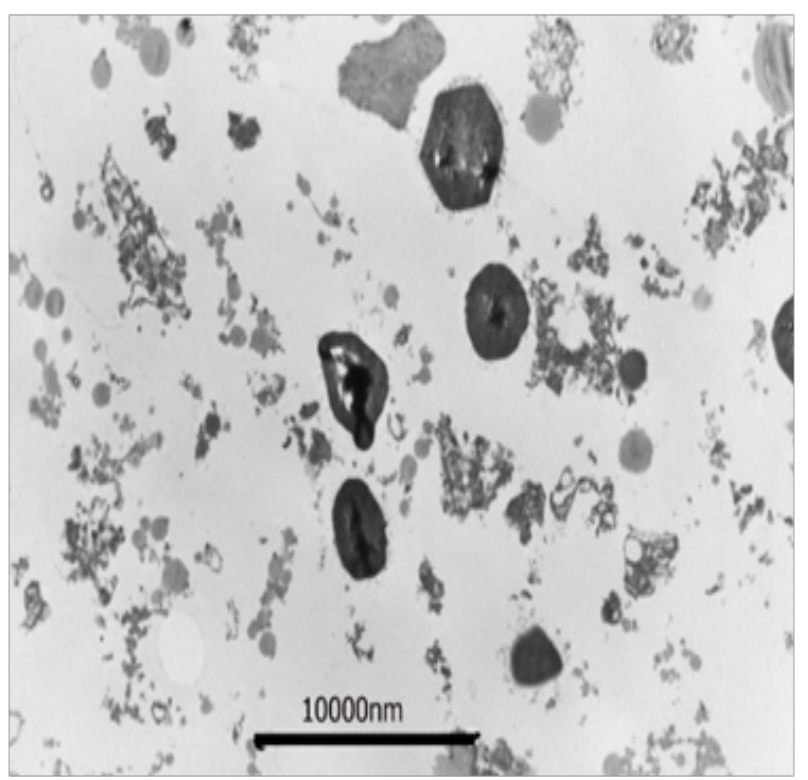

Figure 2 Microphotography of light fraction particles.

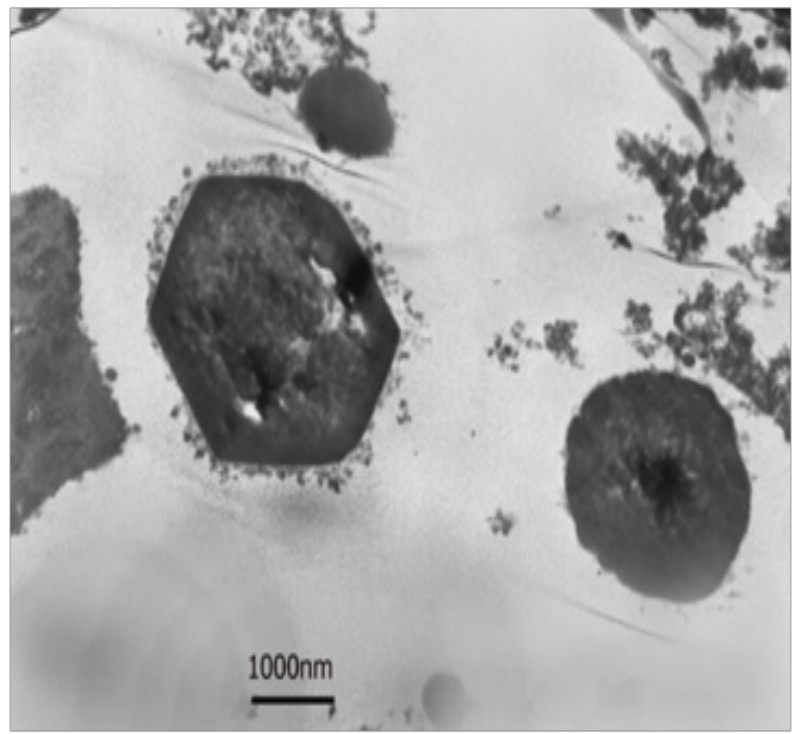

Figure 3 Microphotography of heavy fraction. 


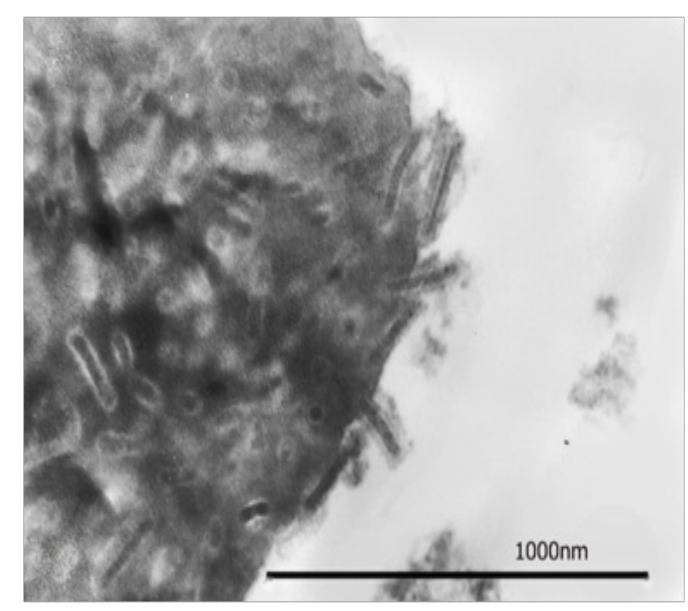

Figure 4 Microphotography of heavy fraction polyhedron at high magnification.

\section{Conclusion}

i. The method developed by us enables us to receive polyhedrons of high purity.

ii. Weakly diseased grain was used for feeding silkworm, which at further feeding and testing will give breeds resistant to the disease nuclear polyhedrose.

\section{Acknowledgements}

None.

\section{Conflict of interest}

The author declares no conflict of interest.

\section{References}

1. Baramidze N, Khutsishvili M, Tskaruashvili Z, et al. Effect of the bluish-green alga Spirulina on mulberry silkworm disease "nuclear polyhedrose". Climate changes and chemicals-the new sericulture challenges. $8^{\text {th }}$ BACSA International Conference; Bulgaria: CLISERI; 2017. p. 97-102.

2. Baramidze N, Khutsishvili M, Tskaruashvili Z, et al. Creation of a mulberry silkworm breed resistant to a disease "nuclear polyhedrose". Climate changes and chemicals-the new sericulture challenges. $8^{\text {th }}$ BACSA International Conference; Bulgaria: CLISERI; 2017. p. 90-92.

3. Baramidze N, Khutsishvili M, Tskaruashvili Z, et al. Prognostics of mulberry silkworm disease-nuclear polyhedrosis in grain phase. Climate changes and chemicals-the new sericulture challenges. $8^{\text {th }}$ BACSA International Conference; Bulgaria: CLISERI; 2017. p. 87-90.

4. Jarvis DL, Bohlmeyer DA, Garcia A. Requirements for nuclear localization and supramolecular assembly of a baculovirus polyhedrin protein. Virology. 1991;185(2):795-810.

5. Eason JE, Hice RH, Johnson JJ, et al. Effects of substituting granulin or a granulin-polyhedrin chimera for polyhedrin on virion occlusion and polyhedral morphology in autographa californica multinucleocapsid nuclear polyhedrosis virus. $J$ Virol. 1998;72(7):6237-6243.

6. Monsma SA, Oomens AG, Blissard GW. The GP64 Envelope fusion protein is an essential baculovirus protein required for cell-to-cell transmission of infection. $J$ Virol. 1996;70(7):4607-4616.

7. Hong T, Summers MD, Braunagel SC. N-terminal sequences from Autographa californica nuclear polyhedrosis virus envelope proteins ODV-E66 and ODV-E25 are sufficient to direct reporter proteins to the nuclear envelope, intranuclear microvesicles and the envelop of occlusion derived virus. Proc Natl Acad Sci U S A. 1997;94(8):4050-4055. 